\title{
Brucellosis and Sacroiliitis: A Common Presentation of an Uncommon Pathogen
}

\author{
James R. Priest, MA, Dennis Low, MD, Cliff Wang, MD, and Thomas Bush, MD
}

Musculoskeletal problems are the most common chief complaint in ambulatory medicine across all specialties, and back pain is one of the top 10 problems encountered by the general practitioner. ${ }^{1,2}$ The differential diagnosis of lower back pain is exhaustive, but a history significant for constitutional symptoms or unusual exposures should prompt a work-up for an infectious cause. We describe the case of a 25-year-old man with a Brucella abortus sacroiliitis and possible orchiitis after consumption of unpasteurized cheese imported from El Salvador. The patient was successfully treated with gentamycin, rifampin, and doxycycline. Though the presentations of brucellosis are myriad, osteoarticular involvement of the axial skeleton is the most common presentation of this zoonotic infection. ${ }^{3}$ In the United States brucellosis is rarely encountered and is typically limited to people who are exposed during travel to endemic areas. Here we review briefly the epidemiology and presentation of a Brucella infection and current recommendations for treatment. (J Am Board Fam Med 2008;21:158-161.)

\section{Case Report}

The patient was a previously healthy 25 -year-old man admitted with a 6-week history of right-sided back and buttock pain, intermittent fever, and nonproductive cough. His pain began after an episode of heavy lifting and was unrelieved by nonsteroidal anti-inflammatory drugs and oral opiates, and progressively worsened over 3 weeks. At the time of admission the patient was able to only ambulate with severe discomfort. The patient did report occasional tactile fevers, night sweats, and weight gain secondary to a lack of physical activity but denied other constitutional and gastrointestinal symptoms. Sexual history was positive for only one partner during the previous year with whom he used barrier protection at all times. This young man did recall ingesting a soft, herbed cheese imported from El Salvador 2 months before the onset of symptoms. He had been seen twice in the emergency department (ED) before admission with similar

This article was externally peer reviewed.

Submitted 19 July 2007; revised 12 October 2007; accepted 17 October 2007.

From Stanford University School of Medicine (JP), Stanford; and the Division of Primary Care (DL, CW) and the Division of Rheumatology (TB), Department of Medicine, Santa Clara Valley Medical Center, San Jose, CA.

Funding: none.

Conflict of interest: none declared.

Corresponding author: James Priest, Stanford University School of Medicine, MSOB, Mail Code 5404, Stanford, CA 94305 (E-mail: jpriest@stanford.edu). complaints of back pain and, during his first visit, of right testicular pain. He was diagnosed with sciatica and treated symptomatically after both earlier encounters.

At the time of admission the patient was afebrile and comfortable at rest, but during movement reported severe pain localized to his right flank. Examination was notable for a $2 / 6$ systolic murmur localized at the right upper sternal border and normal genitourinary findings. He was moderately tender to palpation at the right sacroiliac joint, with pain on straight leg raise, and normal motor strength and reflexes in the lower extremities. Laboratory studies revealed a mildly elevated erythrocyte sedimentation rate of $18 \mathrm{~mm} / \mathrm{hr}$ (normal, 0-15 $\mathrm{mm} / \mathrm{hr}$ ), decreased from $57 \mathrm{~mm} / \mathrm{hr}$ and $30 \mathrm{~mm} / \mathrm{hr}$ at previous ED visits. Antinuclear antibodies, rheumatoid factor, and rapid plasma reagent values were normal. Urine gonnorhea and chlamydia DNA probes were negative. Plain films demonstrated a slight widening of the right sacroiliac joint and magnetic resonance imaging noted enhancement and fluid (Figure 1). Transthoracic echocardiography revealed an absence of vegetations or valvular pathology.

The patient remained intermittently febrile to $100.5^{\circ} \mathrm{F}$. An interventional radiology biopsy of his right sacroiliac joint on hospital day 2 yielded straw colored fluid, few white cells, and Gram-negative 

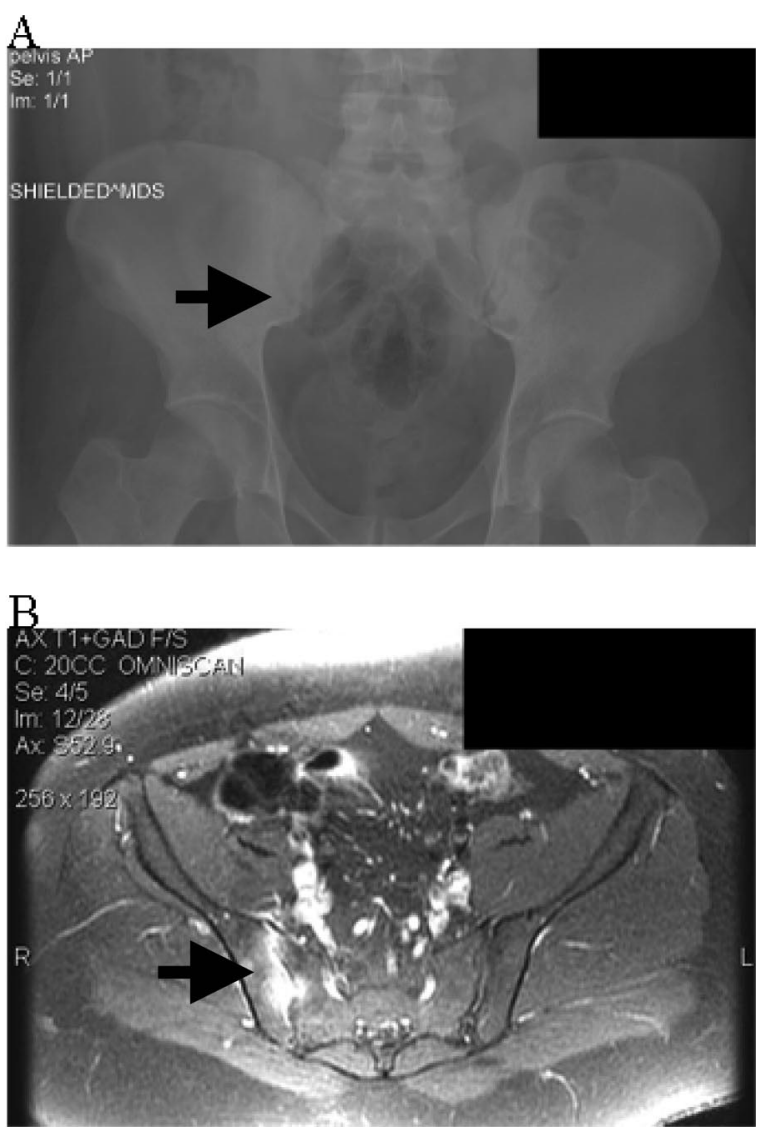

Figure 1. (A) Pelvic plain film shows normal hip joints and subtle widening of the lower portion of the right sacroiliac joint without evidence of erosions or sclerosis. (B) An image from a T1-weighted magnetic resonance imaging series with gadolinium contrast shows enhancement and fluid within the right sacroiliac joint and a normal left sacroiliac joint.

cocco-rods by Gram stain. Serology was positive for Brucella immunoglobulin M, and both fluid and blood culture revealed Brucella species, identified by the California State Laboratory as Brucella abortus. By hospital day 3 his murmur had resolved and, after 7 days of intravenous gentamycin, doxycyline, and rifampin, the patient was afebrile and ambulating without pain. He was discharged on a 6-month course oral rifampin and doxycycline, and at 6 months' follow-up has remained free of symptoms.

\section{Discussion}

We have described an infection of the sacroiliac joint as the focal manifestation of brucellosis that was probably contracted from ingesting unpasteurized dairy products. Osteoarticular involvement of the axial skeleton is the most common presentation of Brucella infection described in the worldwide literature. Infection results from ingestion of as few as 10 microbes of this facultative intracellular pathogen, although there are reports of infection via inhalation and inoculation. ${ }^{4,5}$ Interestingly the Brucella species has evolved complex mechanisms for evasion of the immune system, including protein inhibition of macrophage apoptosis and secretion of a soluble tumor necrosis factor- $\alpha$ inhibitor. ${ }^{6}$ As seen in our patient, the incubation period of brucellosis ranges from weeks to months and the signs, symptoms, and focal sites of infection are numerous and may vary by organism.

The Gram-negative genus Brucella encompasses 7 zoonotic species, of which melitensis, abortus, suis, and, to a lesser degree, canis cause both human and veterinary disease. Infected individuals are typically exposed directly to animal reservoirs or to unpasteurized dairy products prepared from infected animals, which was probably the case with our patient. B. melitensis is the most frequently reported pathogen of the genus worldwide, however Brucellosis is widely considered to be underreported. ${ }^{7,8}$ Most accounts of $B$. melitensis originate from southern Spain, Turkey, and the Persian Gulf region; the largest cohort of patients treated in the United States describes 28 cases of both B. melitensis and $B$. abortus in San Diego since 1979. ' B. suis and canis are infrequently described in the literature.

In endemic regions the infection is prevalent among populations with occupational exposure to livestock; there is serologic evidence of brucellosis in $28.3 \%$ of Saudi farmers and $41.8 \%$ of shepherds. ${ }^{7}$ Estimates of infection for the general population range from $15 \%$ in Saudi Arabia to $4.8 \%$ in Turkey $^{10}$ and $3.4 \%$ in Mexico. ${ }^{11}$ Even in nonendemic regions exposure to livestock considerably elevates the risk of exposure. A survey in the Republic of Ireland showed evidence of exposure in $3.6 \%$ of government agricultural employees, ${ }^{12}$ and in agricultural regions of southern Italy serology suggests a population prevalence of $2.7 \%$ to $3.8 \%{ }^{13}$ Interestingly the Saudi study notes a $13.6 \%$ seropositivity rate among individuals without a history of symptomatic brucellosis, suggesting exposure is widespread in regions of endemic infection. Since the advent of government eradication programs during the 1930s, widespread vaccination of livestock, and pasteurization of dairy products, infection by Brucella in the United States 
has become virtually obsolete, with only 120 cases reported to the Centers for Disease Control and Prevention in 2005. ${ }^{14}$ Brucellosis is still occasionally diagnosed in travelers to endemic areas and consumers of unpasteurized dairy products, with infrequent outbreaks among agricultural workers. A commonly reported risk factor in developed countries appears to be employment as a laboratory worker. ${ }^{15-17}$

The majority of patients infected with Brucella experience fever accompanied by osteoarticular involvement as the predominant focal symptom. Four studies including 757 patients reported that $21 \%$ to $55 \%$ of infected individuals experienced involvement of the bone, most commonly as sacroiliitis or spondylitis. ${ }^{4,9,18,19}$ Neurologic, cardiovascular, obstetric, respiratory, and genitourinary infections have all been reported; however, the most commonly reported site of focal infection in all studies was involvement of the bone. There was no evidence of orchiitis at the time of presentation in our patient, but he had endorsed testicular pain during a previous ED visit.

Serology and culture are required to firmly establish the diagnosis of brucellosis. Commercially available serologic tests cannot specify different species because the antigens cross-react with antibodies to melitensis, abortus, and suis. Although an elevated erythrocyte sedimentation rate is generally suggestive of inflammation or infection, it is only elevated in $50 \%$ of people with brucellosis. ${ }^{20} \mathrm{We}$ did not perform a cell count on the synovial fluid obtained from our patient. However, the overwhelming presence of leukocytes within infected joints is not a common finding in focal brucellosis. ${ }^{21}$ Thus, in the absence of typical markers of infection, a Gram stain and serology of blood or fluid is necessary to determine the presence of Brucella and culture is required to determine the species involved.

For uncomplicated brucellosis without focal involvement a 6-week course of streptomycin and doxycycline is sufficient. ${ }^{22}$ In cases of osteoarticular involvement, recent trials suggest a 6-month course of rifampin, doxycycline, and streptomycin to reduce the elevated incidence of relapse. ${ }^{23,24}$ Relapse occurs in $3.6 \%$ to $4.5 \%$ of patients with uncomplicated brucellosis but is elevated to $10.6 \%$ to $11 \%$ by osteoarticular or focal involvement. ${ }^{18,25,26}$ There is additional evidence for replacing streptomycin with a short course of gentamycin when it is administered in combination with doxycycline; hence our course of treatment. ${ }^{27}$

\section{Conclusions}

We have described a case of Brucella abortus sacroiliitis probably contracted from unpasteurized cheese, which was treated successfully with gentamycin, rifampin, and doxycycline. Osteoarticular involvement of the axial skeleton accompanied by fever is the most common manifestation of brucellosis. Although Brucella is rare in the United States, physicians in areas of the country with large immigrant populations should still be aware of this important clinical entity, which remains endemic in many regions of the world. Serologic evidence suggests widespread exposure in endemic regions and among people with occupational exposure to livestock in countries where the infection has been mostly eradicated. Diagnosis of this reportable zoonosis ideally requires both serology and culture, and treatment should consist of an extended regimen of 2 to 3 antibiotics depending on the presence of focal involvement. When confronted with a patient reporting exposure to livestock, raw dairy products, travel to endemic regions, or employment as a laboratory worker, the primary care physician should consider the clinical presentation, diagnosis, and treatment of Brucella species.

The authors would like to thank Yinyu Tang, MD, and Ivy Lee, MD.

\section{References}

1. Hing E, Cherry DK, Woodwell DA. National Ambulatory Medical Care Survey: 2004 summary. Adv Data 2006;(374):1-33.

2. Middleton KR, Hing E. National Hospital Ambulatory Medical Care Survey: 2004 outpatient department summary. Adv Data 2006;(373):1-27.

3. Gonzalez-Gay MA, Garcia-Porrua C, Ibanez D, Garcia-Pais MJ. Osteoarticular complications of brucellosis in an Atlantic area of Spain. J Rheumatol 1999;26:141-5.

4. Park MY, Lee CS, Choi YS, Park SJ, Lee JS, Lee HB. A sporadic outbreak of human brucellosis in Korea. J Korean Med Sci 2005;20:941-6.

5. Ashford DA, di Pietra J, Lingappa J, et al. Adverse events in humans associated with accidental exposure to the livestock brucellosis vaccine RB51. Vaccine 2004;22:3435-9.

6. Jimenez de Bagues MP, Dudal S, Dornand J, Gross A. Cellular bioterrorism: how Brucella corrupts mac- 
rophage physiology to promote invasion and proliferation. Clin Immunol 2005;114:227-38.

7. Al Sekait M. Seroepidemiological survey of brucellosis antibodies in Saudi Arabia. Ann Saudi Med 1999;19:219-22.

8. Pappas G, Panagopoulou P, Christou L, Akritidis N. Brucella as a biological weapon. Cell Mol Life Sci 2006;63:2229-36.

9. Troy SB, Rickman LS, Davis CE. Brucellosis in San Diego: epidemiology and species-related differences in acute clinical presentations. Medicine (Baltimore) 2005;84:174-87.

10. Cetinkaya Z, Aktepe OC, Ciftci IH, Demirel R. Seroprevalence of human brucellosis in a rural area of Western Anatolia, Turkey. J Health Popul Nutr 2005;23:137-41.

11. Lopez-Merino A, Migranas-Ortiz R, Perez-Miravete A, et al. [Seroepidemiology of brucellosis in Mexico]. Salud Publica Mex 1992;34:230-40.

12. Reid AJ. Brucellosis a persistent occupational hazard in Ireland. Int J Occup Environ Health 2005;11: 302-4.

13. Torre I, Ribera G, Pavia M, Angelillo IF. A seroepidemiologic survey on brucellosis antibodies in southern Italy. Infection 1997;25:150-3.

14. McNabb SJ, Jajosky RA, Hall-Baker PA, et al. Summary of notifiable diseases United States, 2005. MMWR Morb Mortal Wkly Rep 2007;54:1-92.

15. Al Dahouk S, Nockler K, Hensel A, et al. Human brucellosis in a nonendemic country: a report from Germany, 2002 and 2003. Eur J Clin Microbiol Infect Dis 2005;24:450-6.

16. Bouza E, Sanchez-Carrillo C, Hernangomez S, Gonzalez MJ. Laboratory-acquired brucellosis: a Spanish national survey. J Hosp Infect 2005;61:80-3.

17. Noviello S, Gallo R, Kelly M, et al. Laboratoryacquired brucellosis. Emerg Infect Dis 2004;10: 1848-50.
18. Colmenero JD, Reguera JM, Martos F, et al. Complications associated with Brucella melitensis infection: a study of 530 cases. Medicine (Baltimore) 1996;75:195-211.

19. Dokuzoguz B, Ergonul O, Baykam N, et al. Characteristics of $B$. melitensis versus $B$. abortus bacteraemias. J Infect 2005;50:41-5.

20. Pourbagher MA, Pourbagher A, Savas L, et al. Clinical pattern and abdominal sonographic findings in 251 cases of brucellosis in southern Turkey. AJR AJR 2006;187:W191-4.

21. Press J, Peled N, Buskila D, Yagupsky P. Leukocyte count in the synovial fluid of children with cultureproven brucellar arthritis. Clin Rheumatol 2002;21: 191-3.

22. Ersoy Y, Sonmez E, Tevfik MR, But AD. Comparison of three different combination therapies in the treatment of human brucellosis. Trop Doct 2005;35: 210-2.

23. El Miedany YM, El Gaafary M, Baddour M, Ahmed I. Human brucellosis: do we need to revise our therapeutic policy?. J Rheumatol 2003;30:2666-72.

24. Bayindir Y, Sonmez E, Aladag A, Buyukberber N. Comparison of five antimicrobial regimens for the treatment of brucellar spondylitis: a prospective, randomized study. J Chemother 2003;15:466-71.

25. Solera J, Lozano E, Martinez-Alfaro E, Espinosa A, Castillejos ML, Abad L. Brucellar spondylitis: review of 35 cases and literature survey. Clin Infect Dis 1999;29:1440-9.

26. Solera J, Martinez-Alfaro E, Espinosa A, Castillejos ML, Geijo P, Rodriguez-Zapata M. Multivariate model for predicting relapse in human brucellosis. J Infect 1998;36:85-92.

27. Hasanjani Roushan MR, Mohraz M, Hajiahmadi M, Ramzani A, Valayati AA. Efficacy of gentamicin plus doxycycline versus streptomycin plus doxycycline in the treatment of brucellosis in humans. Clin Infect Dis 2006;42:1075-80. 